\title{
Reduction Formulas for Multiple Series
}

\author{
By M. L. Glasser
}

Abstract. A simple procedure is given for reducing broad classes of multiple series to single series. Examples are given for double series.

Suppose that $A_{1} A_{2}=A_{3}$, where $A_{i}$ is a function of $u$ and possesses a series expan$\operatorname{sion} A_{i}=\sum_{n} \phi_{i}(n, u)$. Then we have

$$
\sum_{m, n} \phi_{1}(n, u) \phi_{2}(m, u)=\sum_{n} \phi_{3}(n, u) .
$$

If both sides of (1) are multiplied by some function $f(u)$ and integrated over $u$, we shall have formally

$$
\sum_{m, n} F_{1}(m, n)=\sum_{n} F_{2}(n)
$$

This rather trivial procedure can lead to some remarkable and useful results, as we shall illustrate by some examples.

If $f$ and $g$ are two analytic functions, then, upon multiplication of their Taylor series, we obtain

$$
\sum_{m, n=0}^{\infty} \frac{f^{(n)}(0) g^{(m)}(0)}{m ! n !} F(m+n+1)=\sum_{n=0}^{\infty} \frac{(f g)^{(n)}(0)}{n !} F(n+1),
$$

where $F$ is any Mellin transform.

From the theory of elliptic functions [1], we have the Fourier series

(a) $\operatorname{cn}(2 K x / \pi)=(2 \pi / k K) \sum_{0}^{\infty} q^{(n+1 / 2)}\left(1+q^{2 n+1}\right)^{-1} \cos (2 n+1) x$,

(b) $(2 K / \pi) \operatorname{dn}(2 K x / \pi)=1+4 \sum_{1}^{\infty} q^{n}\left(1+q^{2 n}\right)^{-1} \cos 2 n x$,

$$
(2 K / \pi) \operatorname{cn}(2 K x / \pi) \operatorname{dn}(2 K x / \pi)
$$

(c)

$$
=(2 \pi / k K) \sum_{0}^{\infty}(2 n+1) q^{n+1 / 2}\left(1-q^{2 n+1}\right)^{-1} \cos (2 n+1) x,
$$

where $q=e^{-\pi K^{\prime} / K}$. If we now let $K^{\prime} / K=(2 u / \pi)$, we find on multiplication of (4(a)) by (4(b)) that

$$
\sum_{m, n=-\infty}^{\infty} \frac{\cos (2 m+2 n+1) x}{\cosh (2 m+1) u \cosh 2 n u}=2 \sum_{n=0}^{\infty} \frac{(2 n+1) \cos (2 n+1) x}{\sinh (2 n+1) u},
$$

Received September 8, 1972.

AMS (MOS) subject classifications (1970). Primary 40B05.

Key words and phrases. Multiple series, reduction formula. 
where the addition theorem and evenness for the cosine have been used to simplify the left-hand side.

Next, we multiply both sides of (5) by some summable function $f(x)$, with cosine transform $F(y)$, and integrate over $x$ between the limits 0 and $\infty$. Thus, we have

$$
\sum_{m, n=-\infty}^{\infty} \frac{F(|2 m+2 n+1|)}{\cosh (2 m+1) u \cosh 2 n u}=2 \sum_{n=0}^{\infty} \frac{(2 n+1) F(2 n+1)}{\sinh (2 n+1) u} .
$$

This remarkable result is valid for any summable function $F(x)$.

For example, consider $F_{k}(x)=1$ for $\left(2 k+\frac{3}{2}\right)>|x|, 0$ otherwise. Denoting the sum on the left-hand side of (6) by $S_{k}$, we find that it can be written

$$
S_{k}=s_{0}+s_{1}+\cdots+s_{k},
$$

where

$$
s_{k}=4 \sum_{n=-\infty}^{\infty}[\cosh (4 n+2 k+1) u+\cosh (2 k+1) u]^{-1} .
$$

On the other hand, the sum on the right-hand side of (6) is finite and we have

$$
S_{k}=2[\operatorname{csch} u+3 \operatorname{csch} 3 u+\cdots+(2 k+1) \operatorname{csch}(2 k+1) u] .
$$

Therefore, $s_{k}=S_{k}-S_{k-1}=2(2 k+1) \operatorname{csch}(2 k+1) u$ and, hence,

$$
\begin{aligned}
& \sum_{n=1}^{\infty}[\cosh 2 n u+\cosh (2 k+1) u]^{-1} \\
& \quad=\frac{1}{2}\left[(2 k+1) \operatorname{csch}(2 k+1) u-\frac{1}{2} \operatorname{sech}^{2}\left(k+\frac{1}{2}\right) u\right], \quad k=0,1,2, \ldots .
\end{aligned}
$$

In a similar way, we can derive

$$
\begin{aligned}
\sum_{m, n=-\infty}^{\infty} \frac{F(m+n+1)+F(m-n)}{\sinh (2 m+1) u \cosh (2 n+1) u} & =8 \sum_{n=1}^{\infty} \frac{n F(n)}{\cosh (2 n u)}, \\
\sum_{k, m, n=-\infty}^{\infty} \frac{F(k+m+n+1)+F(k-m+n)}{\cosh (2 k u) \cosh (2 m+1) u \sinh (2 n+1) u} & =8 \sum_{n=1}^{\infty} \frac{n^{2} F(n)}{\sinh (2 n u)},
\end{aligned}
$$

where $F$ is any sine transform (and hence odd).

Thus, taking $F(1)=-F(-1)=1, F(n)=0, n \neq 1$, in (12), we obtain the interesting double series

$$
\sum_{k, n=-\infty}^{\infty} \frac{\sinh [2(k+n)+1] u}{\cosh 2 k u \sinh (2 n+1) u[\cosh 2(2 k+2 n+1) u+\cosh 4 u]}=\operatorname{csch}^{2} 2 u
$$

Battelle Memorial Institute

505 King Avenue

Columbus, Ohio 43201

1. E. T. Whittaker \& G. N. Watson. A Course of Modern Analysis, 4th edition, Cambridge Univ. Press, New York, 1962, p. 510. 